\title{
Determinantes da participação dos domicílios em acordos de pesca no Baixo Amazonas, estado do Pará
}

\section{Drivers of household participation in fishing accords in the Low Amazon region of Pará state, Brazil}

\author{
Stella Zucchetti Schons 1
}

\begin{abstract}
Resumo: Este artigo tem por objetivo discutir diferentes fatores que contribuem para a tomada de decisão de domicílios da várzea do baixo rio Amazonas, no Pará, de participar ou não dos esforços de implementação de acordos de pesca comunitários. Como resultado da aplicação de um modelo econométrico Probit a dados coletados junto a cerca de 650 domicílios em dois momentos do pulso das águas do rio Amazonas, tem-se que maiores valores do benefício do seguro defeso e históricos mais longos de participação nos acordos de pesca contribuem para a decisão de participação nesses acordos. Além disso, a produtividade na atividade da pesca, dentre outros fatores demográficos e exógenos, também contribui para aquela decisão. Esses resultados indicam a relevância do papel do governo e do compromisso moral com a comunidade, assim como o potencial benéfico da assistência técnica de qualidade para o sistema de comanejo da pesca da região.
\end{abstract}

Palavras-chave: incentivos, comanejo, pesca, acordos de pesca.

\begin{abstract}
The objective in this article is to study the different factors contributing to fisher household's decision to participate in community fishing accords in the Low Amazon region of the State of Pará, Brazil. Through the estimation of a weighted Probit model applied to data collected from approximately 650 households in two moments of the Amazon river flood pulse, I found that higher benefits from the defeso insurance and longer household histories of participation in fishing accords contribute positively to the probability of participation in these accords. Moreover, the household fishing productivity, among other factors, also contributes positively to that participation decision. These results suggest the relevance of the role of the government and of the moral commitment with the community as well as the potential benefit of quality technical assistance to the fisheries co-management system in the region.
\end{abstract}

Keywords: incentives, co-management, fisheries, fishing accords.

\section{Introdução}

A gestão de recursos naturais de uso comum vem conquistando espaço nas políticas públicas há mais de 30 anos em todo o mundo, na medida em que o problema

\footnotetext{
${ }^{1}$ Ph.D. in Forestry 2017, Virginia Polytechnic Institute and State University; Master in Environmental Management e Master in Business Administration 2010, Yale University; Bacharel em Ciências Econômicas 2005, Universidade Estadual de Campinas (UNICAMP). Professora Assistente, Department of Forest Resources and Environmental Conservation, Virginia Polytechnic Institute and State University. E-mail: szschons@vt.edu
} 
da exaustão desses recursos coloca em cheque as atividades e os modos de vida das populações que deles dependem (SCHLAGER, 2004). Compreender os incentivos e os determinantes da decisão individual de participar ou não em esquemas de gestão de bens de uso comum é tão importante quanto o entendimento sobre os fatores institucionais necessários para o sucesso desses esquemas (POMEROY et al., 2001; SCHONS, 2017). No contexto dos recursos pesqueiros, este artigo tem por objetivo discutir diferentes fatores que contribuem para a decisão de domicílios da várzea do baixo rio Amazonas, no Pará, de participar dos esforços de implementação de acordos de pesca comunitários.

Bem de uso comum é aquele recurso, natural ou não, cuja exclusão de potenciais beneficiários é custosa, embora possível (GARDNER et al., 1990). Ao mesmo tempo, esse recurso é "rival", ou seja, o consumo de uma unidade dele por um beneficiário significa que outro beneficiário não poderá usufruir daquela mesma unidade. O resultado disso é a exaustão do recurso, uma vez que, enquanto haja oportunidade de se beneficiar dele, alguém buscará capturar esse benefício, seguindo o raciocínio de que ele será capturado de qualquer maneira por outro alguém (HARDIN, 1968; GORDON, 1954). Na busca pela satisfação do interesse individual, o grupo todo de beneficiários do recurso acaba por perdê-lo, como exemplificado na "Tragédia dos Bens Comuns" (HARDIN, 1968).

Em resposta às previsões da "Tragédia dos Bens Comuns" e aos efeitos adversos da consequente onda de gestão centralizada de recursos naturais que tomou conta principalmente do mundo em desenvolvimento entre as décadas de 1960 e 1980, todo um novo campo de pesquisa emanou das análises baseadas em estudos de caso de gestão de bens de uso comum como os elucidados por Elinor Ostrom (SCHLAGER, 2004). O corpo de conhecimento gerado a partir de Ostrom (1990) tem como resultado uma série de condições, na maior parte de natureza institucional, para que sistemas de gestão de bens de uso comum liderados por comunidades surjam e sejam bem-sucedidos (SCHLAGER, 2004; CASTELLO et al., 2009).

Recentemente, a teoria de Ostrom levou ao desenvolvimento do conceito de cogestão, comanejo ou gestão participativa (OVIEDO et al., 2015), uma abordagem que 
tem sido amplamente aceita pelos gestores de recursos pesqueiros, especialmente quando se trata de pesca artesanal de pequena escala. No comanejo, os usuários do recurso compartilham a responsabilidade e o poder sobre ele com o governo, e todos os stakeholders interessados são envolvidos, na busca da promoção de um processo de decisão compartilhado em que as tradições locais, a sustentabilidade, a eficiência e a igualdade sejam valorizados (EVANS et al., 2011; GUTIERREZ et al., 2011). Ou seja, a solução para reduzir a pressão sobre o bem de uso comum é específica ao local e aos seus usuários.

Comanejo é o sistema de gestão compartilhada do recurso pesqueiro que encontramos nas comunidades ribeirinhas da região de Santarém, no estado do Pará. Localmente, esse sistema toma o formato de acordos de pesca (Oviedo et al., 2015), que seriam as unidades de discussão e definição de regras de uso do recurso pesqueiro nos lagos de várzea, onde espécies importantes para a nutrição e renda das comunidades encontram-se vulneráveis à superexploração, principalmente nas épocas de seca (OVIEDO et al., 2015, SILVA e FERREIRA, 2018). Recentemente, os acordos de pesca foram incorporados aos planos de uso dos Projetos de Assentamentos Agroextrativistas (PAEs), na tentativa empreendida pelo Governo Federal de melhor organizar o território da várzea (BENATTI, 2011).

A realidade das famílias que vivem na várzea amazônica é bastante peculiar, uma vez que seu meio de vida é ditado pelo pulso do Rio Amazonas, que alaga as áreas adjacentes ao seu canal principal por aproximadamente seis meses todo ano (BAYLEY e PETRERE, 1989; BAYLEY, 1995). Esse ciclo anual de interação entre rio e terra, associado à água rica em nutrientes que desce dos Andes, resulta num ecossistema de alta produtividade constante e numa variedade de habitats que se renovam a cada ano. $A$ biodiversidade e a diversidade de habitats (lagos, canais etc.) reflete-se na diversidade de atividades produtivas que a população local leva a cabo a fim de extrair a sua subsistência. Trata-se da "estratégia de uso múltiplo de recursos", segundo a qual a família se envolve em mais de uma atividade geradora de renda e nutrição, como a agricultura, criação de gado, produção de mel e hortaliças e pesca, usando diferentes recursos disponíveis no ambiente da várzea (MCGRATH et al., 1993; ALMEIDA, 2006). Fonte principal de proteína para as populações locais, a pesca é uma atividade econômica 
exercida por quase todos os habitantes da várzea. Almeida (2006) descreve que 31\% da renda das famílias na várzea de Santarém provem da atividade da pesca, sendo a segunda maior fonte de renda, atrás apenas das transferências governamentais de renda (aposentadorias e benefícios sociais governamentais, dentre outras).

Quando as populações ribeirinhas se depararam com o aumento da pressão sobre seus recursos pesqueiros devido ao crescimento populacional, nas comunidades e nas grandes cidades da região, e à entrada de barcos frigoríficos provenientes de Santarém, Belém e Manaus, começaram a se organizar dentro de suas comunidades. Esse processo, que contou com o apoio da igreja e de diversas instituições não governamentais, levou à construção dos acordos de pesca a partir dos anos 1980 e evoluiu desde então para o atual sistema de cogestão, que inclui as comunidades e o governo, com diferentes instâncias de negociação. Para maiores detalhes sobre o histórico da formação e funcionamento dos acordos de pesca, veja Mcgrath et al.(1993), Castro et al. (2003), Oviedo et al.(2015) e da Silva e Ferreira (2018).

O funcionamento do sistema de cogestão da pesca na região do Baixo Amazonas, no estado do Pará não se dá sem desafios. A legislação brasileira reconhece todo corpo d'água como bem público (BENATTI, 2011; OVIEDO et al., 2015). Sendo assim, legalmente, é difícil restringir o acesso de pescadores comerciais de fora aos lagos das comunidades que participam dos acordos de pesca. Outro desafio é que o governo encontra dificuldades para fazer a sua parte no comanejo, devido às restrições orçamentárias e de pessoal do Instituto Brasileiro de Meio Ambiente e Recursos Renováveis (IBAMA), agência que representa o governo nesse sistema (OVIEDO et al., 2015). Por outro lado, tem havido episódios de cancelamento e atraso no seguro defeso, uma extensão do sistema de seguridade social e do seguro desemprego no Brasil que visa apoiar os pescadores artesanais durante o período do defeso, quando a pesca é restringida. Este ocorre todo ano na época da desova de espécies em estado de vulnerabilidade. A incerteza do seguro defeso pode deixar as famílias mais vulneráveis à situação de pobreza na várzea que contam com o benefício sem opção, tendo que pescar mesmo quando proibido por lei ou pelos acordos de pesca para manter a subsistência da família. Além disso, algumas comunidades encontram dificuldade de organização e de meios de coibir a violação de acordos de pesca dentro das próprias comunidades. 
O fato é que os incentivos do domicílio que depende da pesca precisam estar alinhados aos objetivos do sistema de gestão proposto - no caso, os acordos de pesca. Mas isso não pode ser realizado sem um entendimento dos fatores que determinam ou, pelo menos, influenciam a decisão do domicílio de participar ativamente nesses sistemas. Assim, a pergunta norteadora neste artigo é: Quais são os fatores que determinam individualmente (em nível domiciliar) a decisão de participar ou não no esforço de implementação de acordos de pesca?

Este artigo visa responder a essas perguntas através de um modelo econométrico, utilizando dados coletados na região do Baixo Amazonas, nas proximidades da cidade de Santarém, no estado do Pará. A hipótese é de que não apenas as características demográficas do domicílio e seu histórico de participação social, mas também a intervenção do governo através do seguro defeso, influenciam a decisão individual (domiciliar) de participar do esforço coletivo de implementação de acordos de pesca.

A literatura de economia que estuda empiricamente os incentivos individuais à participação/cooperação em manejo de recursos naturais de uso comum em contextos de países em desenvolvimento é bastante extensa, mas em sua maior parte tem como foco os recursos florestais. Exemplos dessa literatura incluem Agrawal e Gupta (2005), que analisam a importância de fatores sociais, econômicos e de acesso, além dos benefícios provenientes dos recursos florestais na participação do programa de descentralização da política de gestão florestal na região do Terai, no Nepal. Jumbe e Angelsen (2007) estudam como o nível de dependência das famílias em relação ao recurso florestal influi na participação em programas de comanejo de florestas em Milawai, e encontram o resultado de que em grupos com restrições de acesso a mercados, a experiência do grupo, anos de residência, coesão tribal e pressão social são importantes fatores para a tomada daquela decisão. Mais recentemente, Coulibaly-Lingani et al (2011) analisaram os fatores que influenciam a participação no programa de gestão florestal em Burkina Faso e descobriram que a participação nos processos de decisão da comunidade, a conservação florestal e os benefícios econômicos são os principais objetivos do envolvimento individual no programa, juntamente com o número de pessoas no domicílio, fontes de renda, o status de propriedade da terra e o acesso à assistência técnica. 
Na região Amazônica e para o caso específico dos recursos pesqueiros, as análises do comanejo da pesca têm focado principalmente seus aspectos institucionais (por exemplo, ver MCGRATH et al., 2008). Castello et al. (2009) analisam a importância do envolvimento individual no sistema de manejo na pesca, mas não os incentivos individuais de participação. Mcgrath et al. (2007) reconhecem a importância de entendimento desses incentivos para um melhor desenho desses sistemas. Mas nenhum desses trabaIhos mostra evidências empíricas dos incentivos de participação nos acordos de pesca. Schons (2017) é o único trabalho empírico de que tenho conhecimento que estima os benefícios individuais da participação dos domicílios nos acordos de pesca na mesma área de estudo deste artigo, em termos da economia de tempo que o esforço de implementação dos acordos representa para eles.

Assim, esse artigo contribui para a literatura ao prover a primeira análise empírica sobre os elementos que influenciam a decisão de participação nos esforços de implementação dos acordos de pesca, dentro de um sistema de comanejo de recursos pesqueiros, na região de Santarém. Além disso, até o encerramento deste artigo, não tenho notícia de outra análise dessa natureza que leve em consideração um programa no formato do seguro defeso, de apoio monetário em nível governamental à conservação.

\section{Modelo econométrico}

A teoria econômica referente às decisões domiciliares, de maneira geral, parte do pressuposto de que os domicílios buscam, na sua tomada de decisão sobre como distribuir o tempo entre as diversas atividades produtivas e de lazer, maximizar a utilidade ou os benefícios líquidos (ou seja, benefícios menos custos) extraídos da utilização dos recursos a eles disponíveis (DE JANVRY et al., 1991). Neste artigo, parte-se desse mesmo pressuposto para analisar os fatores que contribuem para a escolha entre participar ou não do esforço de implementação de acordos de pesca.

Se $y_{1}$ é uma variável binária que representa a decisão de participar do esforço de implementação dos acordos de pesca e $U_{e}$ representa a utilidade ou benefício líquido advindo da participação no esforço de implementação dos acordos de pesca por parte 
do domicílio $i$, tem-se que:

$$
y_{i}= \begin{cases}1 & \text { se } U_{e i} \geq 0 \\ 0 & \text { se } U_{e i}<0\end{cases}
$$

Na equação (1) e a utilidade proveniente da decisão de participar dos acordos de pesca for positiva, então o domicílio $i$ participa desse esforço com probabilidade $p_{i}$ e a variável dependente $y_{i}$ será igual a 1 ; se a utilidade for negativa, o domicílio $i$ não participa do esforço de implementação dos acordos de pesca e $y_{i}=0$ com probabilidade $1-p_{i}$. Existem vários elementos que afetam a decisão de participação nesse esforço, mas, em última instância, parte-se aqui do pressuposto de que ela depende da possibilidade de esse esforço se traduzir em melhores condições de vida para os membros do domicílio. Por exemplo, através de maior renda e menor esforço de pesca por unidade de peixe capturado, possibilitado por um recurso pesqueiro de melhor qualidade (CASTELLO et al., 2009). Além disso, os benefícios líquidos provenientes do esforço de participação nos acordos de pesca são comparados com os benefícios líquidos que podem ser obtidos aplicando o mesmo esforço em outras atividades domiciliares - produtivas ou não. Ou seja, o custo de oportunidade do esforço de participação em acordos de pesca também é considerado nos custos do "cálculo" do benefício líquido resultante daquela decisão.

O objetivo é utilizar um modelo Probit para estimar a probabilidade de que um domicílio representativo da várzea de Santarém e região escolha participar no esforço de implementação dos acordos de pesca. Assim,

$$
p_{i} \equiv \operatorname{Pr}\left[y_{i}=1 \mid x\right]=\Phi\left(x^{\prime} \beta\right)=\int_{-\alpha}^{x^{\prime} \beta} \Phi(z) d z
$$

onde $x$ é um vetor de variáveis independentes $x_{i}=\left[x_{i 1} x_{i 2} x_{i j} \ldots x_{i k}\right]$ de dimensões $\mathrm{N}$ X K (N indivíduos $i$ e $\mathrm{K}$ variáveis independentes, discutidas abaixo, que determinam a probabilidade de participar ou não nos acordos de pesca) e $\beta$ é o vetor de dimensões $\mathrm{K} X 1$ que é estimado e cujos efeitos marginais são calculados. $\Phi\left(x^{\prime} \beta\right)$ é a função de distribuição acumulada normal padrão. O modelo Probit neste artigo é estimado através 
do método de máxima verossimilhança, cujas condições de primeira ordem são:

$$
\sum_{i=1}^{N} w_{i}\left(y_{i}-\Phi\left(x_{i}^{\prime} \beta\right)\right) x_{i}=0 .
$$

Na equação (3), o peso $w_{i}=\Phi\left(x^{\prime} \beta\right) /\left[\Phi\left(x^{\prime} \beta\right)\left(1-\Phi\left(x^{\prime} \beta\right)\right)\right]$ varia conforme o ponto considerado na distribuição das diferentes variáveis. O interesse nesse artigo é nos efeitos marginais, ou seja, no efeito que a variação de uma unidade de uma das variáveis independentes tem sobre a probabilidade de o domicílio participar do esforço de implementação dos acordos de pesca $\partial p_{i} / \partial x_{i j}$, que também varia conforme o ponto considerado na distribuição. Para maiores detalhes do modelo Probit, ver (CAMERON e TRIVEDI, 2005).

No que se refere aos fatores que determinam a probabilidade de participação do domicílio no esforço de implementação dos acordos de pesca na sua comunidade, constantes no vetor $x_{i}$ acima, inclui-se uma série de variáveis, constantes na tabela 2 . Dentre elas estão variáveis demográficas, como o número de pessoas que vivem no domicílio, o número de idosos, anos de escolaridade, o número de moradores que trabalham fora das atividades produtivas do domicílio (por exemplo, na cidade, nas escolas etc.) e bens (valor das embarcações que o domicílio possui). Também foram incluídas variáveis exógenas, como o tempo que se leva para chegar ao centro regional (sede do município de Santarém) e ao mercado mais próximo, além dos preços da gasolina, da unidade de melancia (produto agrícola mais comum à época da nossa coleta de dados), da cabeça de gado e o valor da diária de trabalho. Esses preços expressam custos de oportunidade da atividade pesqueira e, por consequência, do tempo gasto no esforço de implementação dos acordos de pesca. A produtividade do domicílio na atividade pesqueira, expressa em termos de $\mathrm{kg}$ de pescado por dia ( $1 \mathrm{dia}=8$ horas de trabalho) foi incluída no modelo, pois pode afetar a decisão de engajamento ativo nos acordos de pesca se esse esforço possibilita menos tempo necessário para pescar uma quantidade fixa de pescado ou, por outro lado, pode significar que o domicílio tem mais tempo para participar do acordos.

As variáveis de maior interesse, no entanto, são o número de anos desde que o domicílio participou pela primeira vez em acordos de pesca (histórico do domicílio com 
os acordos de pesca) e o valor do benefício do seguro defeso no último ano. Por um lado, essas variáveis nos permitem verificar como iniciativas que visam à conservação do recurso pesqueiro em dois diferentes níveis, de comunidade e governamental respectivamente, afetam as decisões domiciliares de participação nos acordos de pesca. Na tentativa de capturar os efeitos da organização social da comunidade para implementar os acordos de pesca, foi incluída a variável binária "existe ou não manejo pesqueiro" (o manejo do pirarucu, por exemplo), como uma proxy da capacidade da comunidade de se organizar. Finalmente, incluímos o número de líderes sociais que vivem no domicílio como proxy para o comprometimento dos seus membros para com os acordos de pesca.

Nos próximos parágrafos, os dados disponíveis para estimar o modelo econométrico acima exposto e os detalhes sobre a forma como foram coletados são discutidos, seguido pela apresentação dos resultados. Finalmente, serão discutidas as implicações desses resultados na formulação de políticas públicas.

\section{Dados}

Os dados para essa pesquisa foram coletados durante duas rodadas de entrevistas realizadas no ano de 2015 em 24 comunidades distribuídas $100 \mathrm{~km}$ rio abaixo e rio acima da cidade de Santarém, no estado do Pará. A tabela 1 mostra a lista das comunidades incluídas na pesquisa e os PAEs onde estão localizadas. A figura 1 mostra 0 mapa da localização e distribuição das entrevistas.

\begin{tabular}{|c|c|c|c|}
\hline Comunidade & PAE & Comunidade & PAE \\
\hline Ipanema, Toco e Torrão & Ilhas Reunidas & Ilha de São Miguel & \multirow{2}{*}{ Aritapera } \\
\hline São Francisco & \multirow{2}{*}{ Madalena } & Água Preta & \\
\hline Costa do Iranduba & & Costa do Tapará & \multirow{7}{*}{ Tapará } \\
\hline Santana do Três Ilhas & \multirow{4}{*}{ Três Ilhas } & Pixuna & \\
\hline Nossa Senhora das Graças & & Tapará Miri & \\
\hline Januária & & Santa Maria & \\
\hline Ilha do Carmo & & Correio & \\
\hline Salvação & \multirow{2}{*}{ Salvação } & Boa vista & \\
\hline Ilha do Carmo & & Tapará Grande & \\
\hline Atumã & \multirow{2}{*}{ Atumã } & Aracampina & Ituqui \\
\hline Centro do Arapiri & & Nova vista & Área de quilombo \\
\hline
\end{tabular}

As comunidades acima apresentam significativa variação nos diversos elementos relevantes para a nossa análise, como o nível de organização comunitária e implemen- 
tação dos acordos de pesca, variação esta imprescindível para a realização da análise. A escolha dos domicílios dentro de cada comunidade foi feita de duas formas, aleatória ou $100 \%$ das casas, conforme as condições de campo.

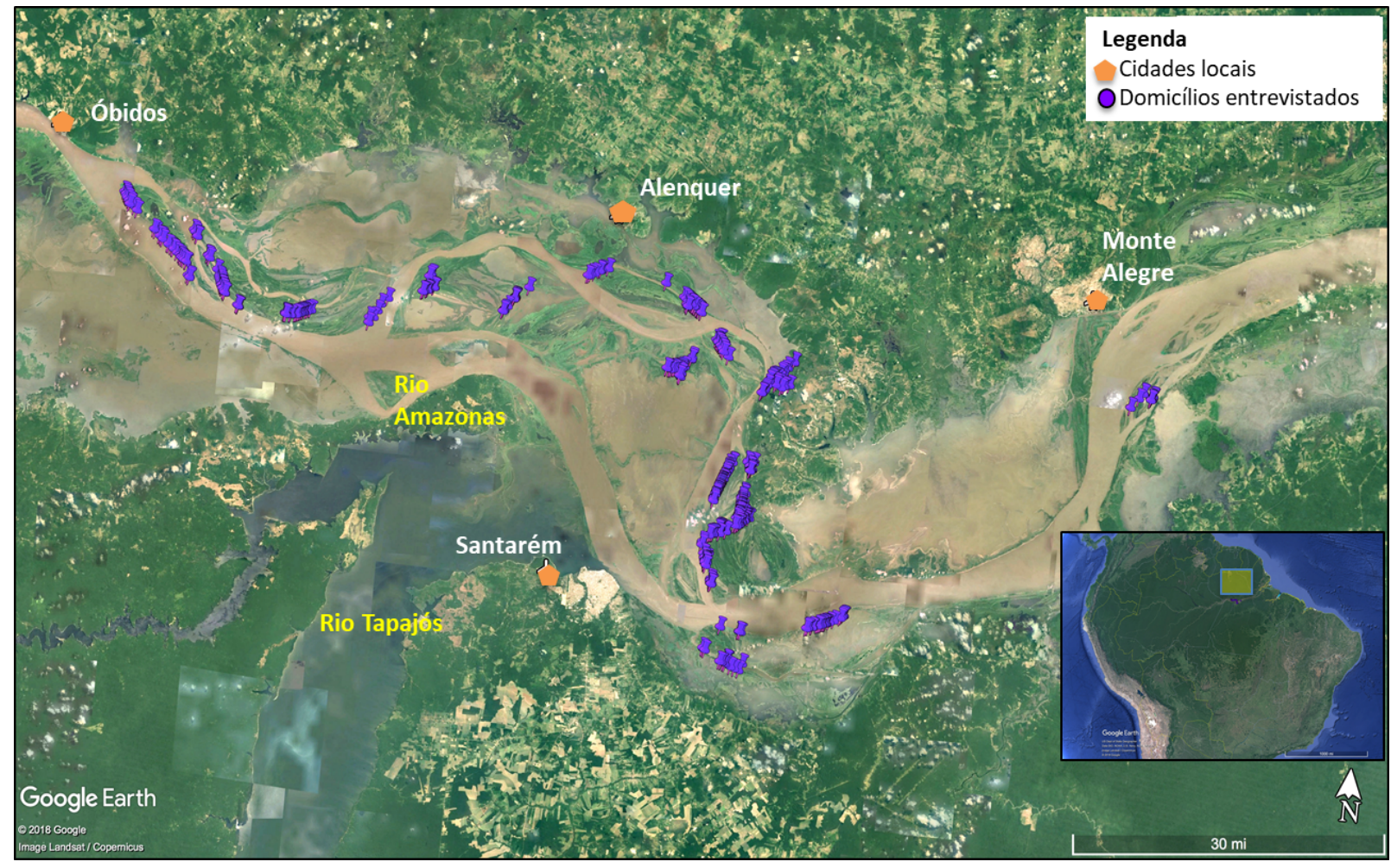

Figura 1. Localização das entrevistas realizadas. Fonte: GoogleEarth. Acesso 27/08/18.

Cada rodada de entrevistas teve duração de aproximadamente 30 dias. Na primeira, realizada no mês de setembro, foram entrevistadas 700 famílias e na segunda, no mês de novembro, foram entrevistadas 644 famílias, totalizando 1344 entrevistas. 0 instrumento de coleta de dados constituiu-se de questionário estruturado com perguntas sobre o domicílio (por exemplo: composição familiar, acesso a infraestrutura, serviços de saúde e educação, acesso a mercados, fontes de renda e atividades econômicas) e, mais detalhadamente, sobre a atividade da pesca. Também foram incluídas perguntas sobre a participação do domicílio em grupos locais e nos acordos de pesca. A maioria das perguntas (a não ser que explicitamente especificado) teve como período de referência os três meses que antecederam imediatamente cada entrevista, de modo que foram considerados os períodos, grosso modo, da cheia e da vazante/seca.

A tabela 2 apresenta as estatísticas descritivas das varáveis incluídas no modelo estimado por grupo de domicílios que participaram e dos domicílios que não participaram 
do esforço de implementação de acordos de pesca na sua comunidade no período coberto pela pesquisa (junho/julho a novembro/dezembro de 2015). Realizou-se um teste $t$ que verifica se há diferenças estatisticamente significantes em cada variável entre esses dois grupos. Considerando o total de todas as entrevistas, na período da cheia, 37\% participaram dos acordos de pesca. Já no período da seca, essa proporção subiu para 55\%. Afirmar que esses domicílios fizeram esforço para implementar os acordos de pesca significa que eles participaram ativamente nesses acordos durante o período de referência, através, por exemplo, de reuniões, do planejamento das atividades e do monitoramento de lagos, entre outras atividades. Considerando que o nível das águas na várzea do Rio Amazonas desce consideravelmente durante a seca, formando os lagos de várzea, é de se esperar que durante essa época ocorra um maior esforço para monitorar locais onde o pescado é mais facilmente capturado.

Algumas variáveis chamam a atenção na tabela 2, sendo significativa a diferença entre as médias do grupo dos domicílios que não implementaram acordos de pesca e do grupo daqueles que participaram desses acordos. Em relação a variáveis demográficas, a média do número de pessoas que vivem no domicílio e a média de mulheres são maiores para o grupo dos domicílios que participam do esforço de implementação dos acordos de pesca. Esse fato sugere que a participação feminina na negociação e engajamento nesses acordos seja relevante, como discutem Silva e Ferreira (2018). Por outro lado, na média o número de idosos é menor para esse grupo. Essas estatísticas sugerem que um maior número de pessoas representa mais trabalho disponível a ser alocado para atividades relacionadas aos acordos de pesca. Já a presença de idosos diminui a disponibilidade de trabalho, o que pode ser explicado pelo fato de ser a pesca uma atividade que requer bastante esforço físico, ou então pela circunstância de o idoso estar aposentado. A idade média do chefe de domicílio é menor, mas a média de anos de escolaridade é maior para o grupo dos que decidiram participar dos acordos de pesca no período de referência. Este último fato pode estar ligado a uma maior conscientização ambiental e melhor entendimento da relevância dos acordos de pesca para a comunidade.

Fatores exógenos, cuja diferença é estatisticamente significativa entre o grupos dos que participaram do esforço de implementação de acordos de pesca e dos que não 
Tabela 2. Estatísticas Descritivas

\begin{tabular}{|c|c|c|c|c|c|c|c|c|}
\hline \multirow[t]{2}{*}{ Variável } & \multicolumn{3}{|c|}{$\begin{array}{l}\text { Esforço de partici- } \\
\text { pação nos acordos } \\
\text { de pesca }=0\end{array}$} & \multicolumn{3}{|c|}{$\begin{array}{l}\text { Esforço de partici- } \\
\text { pação nos acordos } \\
\text { de pesca }=1\end{array}$} & \multirow{2}{*}{\multicolumn{2}{|c|}{$\begin{array}{l}\text { Teste t de } \\
\text { diferença } \\
\text { entre os } \\
\text { grupos }\end{array}$}} \\
\hline & Média & $\mathrm{N}$ & $\mathrm{DP}$ & Média & $\mathrm{N}$ & $\mathrm{DP}$ & & \\
\hline $\begin{array}{l}\text { Benefício do defeso no úl- } \\
\text { timo ano }(\mathrm{R} \$)\end{array}$ & 2.990 & 702 & 2.788 & 4.152 & 590 & 2.499 & $(8,10)$ & $* * *$ \\
\hline Idade chefe de domicílio & 48,17 & 700 & 15,98 & 44,38 & 582 & 12,63 & 4,60 & *** \\
\hline N. pessoas no domicílio & 4,34 & 703 & 2,29 & 4,69 & 590 & 2,08 & $(2,89)$ & $* * *$ \\
\hline N. mulheres & 1,24 & 703 & 0,79 & 1,37 & 590 & 0,86 & $(2,92)$ & 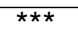 \\
\hline N. crianças & 1,2 & 703 & 1,46 & 1,31 & 590 & 1,34 & $(1,37)$ & \\
\hline N. idosos & 0,34 & 703 & 0,65 & 0,15 & 590 & 0,44 & 6,06 & $* * *$ \\
\hline $\begin{array}{l}\text { N. pessoas que trabalharam } \\
\text { fora do domicílio }\end{array}$ & 30,39 & 703 & 801,38 & 0,47 & 590 & 7,21 & $(1,12)$ & \\
\hline $\begin{array}{l}\text { Tempo desde 1a participa- } \\
\text { ção em acordos de pesca } \\
\text { (anos) }\end{array}$ & 7,10 & 653 & 10,16 & 13,47 & 563 & 9,37 & $-11,29$ & $* \star \star \star$ \\
\hline Escolaridade (anos) & 5,26 & 702 & 3,37 & 5,85 & 586 & 3,28 & $(3,18)$ & $* * *$ \\
\hline Tempo até Santarém (min) & 239 & 654 & 156 & 207 & 583 & 121 & 4,04 & $* \star * \star$ \\
\hline $\begin{array}{l}\text { Tempo até mercado mais } \\
\text { próximo (min) }\end{array}$ & 157 & 701 & 75 & 171 & 589 & 139 & $(2,22)$ & ** \\
\hline N. sítios de pesca & 6,48 & 703 & 0,95 & 6,03 & 590 & 1,06 & 7,92 & $* * *$ \\
\hline $\begin{array}{l}\text { Produtividade na pesca } \\
\text { (Kg/dia) }\end{array}$ & 51,09 & 643 & 341,68 & 86,27 & 566 & 518,05 & $(1,41)$ & \\
\hline $\begin{array}{l}\text { Valor da diária de trabalho } \\
(\mathrm{R} \$)\end{array}$ & 37,36 & 703 & 5,37 & 37,44 & 590 & 5,01 & $(0,27)$ & \\
\hline Preço gasolina $(\mathrm{R} \$ / \mathrm{l})$ & 4,60 & 685 & 0,57 & 4,74 & 585 & 0,55 & $(4,55)$ & ${ }^{* * *}$ \\
\hline Preço melancia (R\$/und) & 5,73 & 703 & 2,44 & 5,31 & 590 & 1,07 & 2,18 & ** \\
\hline $\begin{array}{l}\text { Valor do gado adulto } \\
(\mathrm{R} \$ / \text { cabeça) }\end{array}$ & 1.258 & 703 & 971,69 & 1.231 & 590 & 303,81 & 0,64 & \\
\hline $\begin{array}{l}\text { Nível de conflito na comuni- } \\
\text { dade }(0-3)\end{array}$ & 0,63 & 699 & 1,01 & 0,90 & 583 & 1,04 & $(4,70)$ & 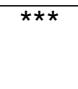 \\
\hline $\begin{array}{l}\text { N. membros do domicílio em } \\
\text { papel de liderança }\end{array}$ & 0,25 & 703 & 2,63 & 0,28 & 588 & 0,60 & $(4,53)$ & 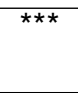 \\
\hline $\begin{array}{l}\text { Valor total das embarcações } \\
(\mathrm{R} \$)\end{array}$ & 5.642 & 703 & 11.296 & 5.714 & 590 & 8.360 & $(0,13)$ & \\
\hline Toda/parte da pesca em rios & 0,32 & 227 & - & 0,36 & 215 & - & & \\
\hline $\begin{array}{l}\text { Há manejo da pesca na co- } \\
\text { munidade }\end{array}$ & 0,34 & 238 & & 0,48 & 283 & & & \\
\hline
\end{tabular}

Nível de significância: ${ }^{* * *} p<0,01 ;{ }^{* *} p<0,05 ;{ }^{*} p<0,10$ 
participaram são a distância média ou tempo médio para se deslocar do domicílio até Santarém (menor para o grupo que participou dos acordos de pesca) e o tempo que levou para chegar ao mercado mais próximo (maior para o grupo que participou dos acordos). A proximidade de Santarém, onde estão localizadas agências de governo e organizações não governamentais que incentivam os acordos de pesca pode ser um fator que contribui para a decisão de participar em acordos de pesca. Os preços médios da gasolina e da melancia pagos pelos dois grupos também são estatisticamente diferentes, o que sugere que o custo de oportunidade da atividade pesqueira na produção agrícola e o custo dos combustíveis podem influenciar na decisão de participar ativamente dos acordos de pesca.

A média do valor total recebido pelo domicílio em seguro defeso é bastante diferente entre o grupo dos que participaram e dos que não participaram em acordos de pesca, sendo a média do primeiro grupo $R \$ 1,162$ maior do que este último. Ou seja, provavelmente, o benefício do seguro defeso influencie na decisão de participar do esforço de implementação dos acordos de pesca, no espírito de conservação do recurso pesqueiro dessa política pública - e porque o benefício relaxa a restrição orçamentária do domicílio, liberando recursos, ou seja, tempo, para a realização de atividades ligadas aos acordos de pesca. A média de tempo transcorrido desde que o domicílio se engajou pela primeira vez em acordos de pesca é cerca de seis anos maior para o grupo de domicílios que participou dos acordos de pesca nos últimos meses. A média do número de líderes sociais é também maior para este grupo assim como o nível percebido de conflito na comunidade, ambas variáveis que sugerem comprometimentos desse grupo de famílias e maior movimentação social em meio a negociações e à implementação dos acordos. A seguir, os resultados do modelo econométrico Probit estimado são apresentados.

\section{Resultados}

O modelo Probit foi estimado utilizando os dados coletados em campo apresentados e discutidos acima e o pacote estatístico STATA (CAMERON e TRIVEDI, 2010). A fim de evitar problemas de heterocedasticidade nas diferenças de métodos utilizados para coletar os dados de uma comunidade para a outra, ponderou-se cada observação pelo 
inverso da proporção de entrevistados em relação à população total da comunidade $\mathrm{2}^{2}$. $\mathrm{A}$ tabela 3 apresenta os efeitos marginais médios calculados a partir das estimativas dos coeficientes do modelo (estes podem ser disponibilizados mediante comunicação com a autora).

Vale lembrar que, através do modelo Probit, busca-se estimar a contribuição marginal das diferentes variáveis para a probabilidade $p_{i}$ de o domicílio tomar a decisão de participar ou não no esforço de implementação dos acordos de pesca da sua comunidade. O modelo estimado acima inclui uma variável binária ("Rodada") que considera o momento em que cada entrevista foi realizada (cheia $=1$; seca $=0$ ). Também inclui-se a elevação do terreno do domicílio e variáveis binárias para indicar o PAE onde se encontra cada domicílio a fim de considerar no modelo diferenças físicas Também inclui-se a elevação do terreno do domicílio e variáveis binárias para indicar o PAE onde se encontra cada domicílio a fim de considerar no modelo diferenças físicas e de organização social que possam afetar de alguma forma as decisões dos seus membros.

Cada efeito marginal médio apresentado na tabela 3 refere-se à média dos efeitos marginais individuais de toda a amostra sobre a probabilidade de tomar a decisão de participar nos esforços dos acordos de pesca. Como mencionado acima, no entanto, esses efeitos marginais variam conforme o locus na distribuição de cada variável. Esses efeitos marginais médios nos possibilitam verificar: (a) as variáveis relevantes para a decisão de participar nos acordos; e (b) o sentido do efeito (ou seja, se a variável contribui positiva ou negativamente para a decisão de participação ativa nos acordos de pesca pelo domicílio).

Dentre a série de variáveis que são significativas mas com efeito negativo sobre a decisão de participação em acordos de pesca por parte do domicílio tem-se o nível das águas na várzea no momento de decidir sobre a participação ou não. Ou seja, na época em que as águas do rio estão mais altas e há inundação, é menor a probabilidade de participação em acordos de pesca - 19\% menor em média, o que é esperado pois é na época da seca que existe uma maior preocupação com relação à vulnerabilidade do pescado nos lagos de várzea (OVIEDO et al., 2015; SILVA e FERREIRA, 2018).

\footnotetext{
${ }^{2}$ As versões do modelo Probit com erros robustos e com correção utilizando bootstrap resultaram em estimativas similares aos apresentados neste artigo e são significativos.
} 
Em relação às variáveis demográficas, a idade do chefe do domicílio e o número de membros idosos atingem um nível de significância de 10\% e contribuem negativamente para a decisão do domicílio de participar dos acordos de pesca: a probabilidade de participação reduz-se em média 0,03\% por ano de vida a mais do chefe de domicílio e 6,5\% para cada aposentado no domicílio, respectivamente. Seja pelo esforço físico da atividade da pesca, que pode ser extenuante para os idosos, seja pela aposentadoria, o fato é que o domicílio pode contar menos com o trabalho desses membros. Já o número de membros trabalhando fora das atividades do domicílio tem efeito marginal médio positivo de $0,04 \%$ para cada membro trabalhando fora sobre a decisão de participar dos acordos de pesca. Esse efeito provavelmente deve-se a um relaxamento na restrição orçamentária do domicílio.

Outras variáveis exógenas, sobre as quais o domicílio não tem controle e que afetam a probabilidade de participação nos acordos de pesca são o preço da melancia e o valor do gado, com efeito marginal médio de 3,5\% e 0,01\% para cada real a mais no preço da melancia e da cabeça de gado, respectivamente. Essas variáveis expressam a importância do custo de oportunidade da atividade agrícola e pecuária para os acordos de pesca e a estratégia de uso de recursos múltiplos (MCGRATH et al., 2007), pois quando aumenta o preço de mercado de outros produtos que o domicílio produz, menos tempo é gasto na implementação dos acordos de pesca. Curiosamente, o preço da gasolina, que é o combustível utilizado nos motores de rabeta que a maioria dos domicílios entrevistados possuem, tem efeito positivo sobre a probabilidade de participação nos acordos de pesca. Esse aspecto merece maior estudo, tendo em vista que o monitoramento de lagos pode consumir uma quantidade considerável de combustível (OVIEDO et al., 2015). Já o valor dos bens representado pelo valor das embarcações que o domicílio possui tem efeito negativo $(0,02 \%$ para cada real do valor da embarcação) sobre a probabilidade de participação do domicílio nos acordos de pesca, o que pode indicar que aqueles domicílios que possuem maior número de embarcações ou embarcações maiores (barco ou voadeira mais potente) estão envolvidos em outras atividades, como o transporte de pessoas ou de mercadorias e, assim, têm menos disponibilidade para participar dos acordos.

Algumas variáveis referentes especificamente à atividade da pesca são altamente 
Tabela 3. Modelo Probit Estimado - Efeitos Marginais Médios

Variável dependente: O domicílio participou do esforço de implementação de acordos de pesca nos três meses antes da entrevista? $(\mathrm{Sim}=1$; Não=0)

\begin{tabular}{|c|c|c|c|c|}
\hline Variáveis independentes: & $\begin{array}{l}\text { Efeito Marginal } \\
\text { Médio }(\mathrm{dy} / \mathrm{dx})\end{array}$ & Erro Padrão & \multicolumn{2}{|l|}{$\bar{z}$} \\
\hline Rodada de entrevistas $(1=1 \mathrm{a} ; 0=2 \mathrm{a})$ & $(0,19340)$ & 0,03346 & $(5,78)$ & *** \\
\hline Elevação da casa (pés) & 0,00099 & 0,00053 & 1,87 & * \\
\hline Benefício do defeso no último ano $(\mathrm{R} \$)$ & 0,00004 & 0,00001 & 3,26 & *** \\
\hline Idade do/da chefe de domicílio & $(0,00380)$ & 0,00220 & $(1,72)$ & * \\
\hline N. de pessoas no domicílio & 0,02162 & 0,01830 & 1,18 & \\
\hline N. de mulheres & $(0,00667)$ & 0,02591 & $(0,26)$ & \\
\hline N. de crianças & $(0,00735)$ & 0,02381 & $(0,31)$ & \\
\hline N. de idosos & $(0,06455)$ & 0,03382 & $(1,91)$ & * \\
\hline $\begin{array}{l}\text { N. de pessoas que trabalham fora do domicí- } \\
\text { lio }\end{array}$ & 0,00417 & 0,00179 & 2,33 & ** \\
\hline $\begin{array}{l}\text { Tempo desde a primeira participação em } \\
\text { acordos de pesca (anos) }\end{array}$ & 0,01800 & 0,00363 & 4,95 & 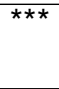 \\
\hline Escolaridade (anos) & $(0,00164)$ & 0,00494 & $(0,33)$ & \\
\hline Tempo até Santarém (minutos) & 0,00007 & 0,00029 & 0,26 & \\
\hline Tempo até mercado mais próximo (minutos) & $(0,00009)$ & 0,00017 & $(0,53)$ & \\
\hline N. de sítios de pesca & $(0,09754)$ & 0,02059 & $(4,74)$ & *** \\
\hline Produtividade na pesca $(\mathrm{Kg} / \mathrm{dia})$ & 0,00009 & 0,00004 & 2,22 & ** \\
\hline $\begin{array}{l}\text { Valor da diária de trabalho na comunidade } \\
(\mathrm{R} \$)\end{array}$ & 0,00248 & 0,00365 & 0,68 & \\
\hline Preço gasolina (R $\$ /$ litro) & 0,09506 & 0,04218 & 2,25 & ** \\
\hline Preço melancia ( $\mathrm{R} \$ /$ unidade) & $(0,03484)$ & 0,01880 & $(1,85)$ & * \\
\hline Valor do gado ( $\mathrm{R} \$ /$ cabeça) & $(0,00006)$ & 0,00003 & $(2,27)$ & ** \\
\hline Nível de conflito na comunidade (0 a 3) & 0,05674 & 0,02461 & 2,31 & ** \\
\hline $\begin{array}{l}\text { Parte ou toda a pesca praticada pelo domicí- } \\
\text { lio em rios }(\operatorname{Sim}=1 ; \mathrm{Não}=0)\end{array}$ & $(0,01670)$ & 0,03934 & $(0,42)$ & \\
\hline $\begin{array}{l}\text { Há manejo pesqueiro na comunidade } \\
(\mathrm{Sim}=1 ; \mathrm{Não}=0)\end{array}$ & 0,05325 & 0,03451 & 1,54 & \\
\hline $\begin{array}{l}\text { N. de membros do domicílio em papel de li- } \\
\text { derança na comunidade }\end{array}$ & 0,04960 & 0,04517 & 1,10 & \\
\hline $\begin{array}{l}\text { Valor total das embarcações do domicílio } \\
(\mathrm{R} \$)\end{array}$ & $(0,00000)$ & 0,00000 & $(2,28)$ & *** \\
\hline
\end{tabular}

Nível de significância: ${ }^{* * *} p<0,01 ;{ }^{* *} p<0,05 ;{ }^{*} p<0,10$

significativas (com nível de significância de 5\% e 1\%). O número de sítios de pesca visitados no período de referência afeta negativamente a probabilidade de participação do domicílio nos acordos de pesca. Segundo o modelo estimado, para cada local de pesca a mais, a probabilidade de participação do domicílio é reduzida em 9,8\%. Por outro lado, a produtividade do domicílio na atividade pesqueira (volume em $\mathrm{kg}$ pescado por dia de trabalho) afeta positivamente a decisão de participar nos acordos: $0,01 \%$ de probabilidade de participação para cada kg a mais de pescado por dia de trabalho. Maior produtividade significa menos tempo necessário para pescar o necessário para satisfazer as necessidades nutricionais e de renda do domicílio, e mais tempo para atividades como 
o envolvimento nos acordos de pesca. Curiosamente, a percepção de um nível de conflito mais alto na comunidade está relacionado com uma maior probabilidade de participação nos acordos de pesca. Por um lado, esperar-se-ia que um nível mais alto de conflito afastasse os domicílios do envolvimento nos acordos. Por outro lado, o nível mais alto de conflito pode indicar a maior necessidade de negociação de regras e monitoramento dos locais de pesca e também maior comprometimento do domicílio.

Voltando para as variáveis que indicam instrumentos de gestão do recurso pesqueiro na região (um em nível governamental federal e outro em nível de comunidade), tanto o valor do benefício recebido pelo domicílio através do programa seguro defeso quanto o tempo transcorrido, em anos, desde o seu primeiro envolvimento nos acordos de pesca são significativas e possuem efeito marginal médio positivo sobre a decisão de participação nos esforços dos acordos de pesca: 0,004\% para cada real a mais de benefício do seguro defeso e 1,8\% para cada ano a mais de envolvimento nos acordos de pesca. Ou seja, o seguro defeso parece gerar o efeito desejável de contribuir para que o domicílio de pescadores se esforce mais para participar em ações que visem à redução da pressão sobre o recurso pesqueiro na região do Baixo Amazonas. Ao mesmo tempo, o modelo indica que o histórico de envolvimento do domicílio nos acordos de pesca interfere na decisão de participação, o que pode ser reflexo da extensão do tempo no trabalho da comunidade e do compromisso moral ou pressão social que há para a participação do domicílio nos acordos de pesca (POMEROY et al., 2001).

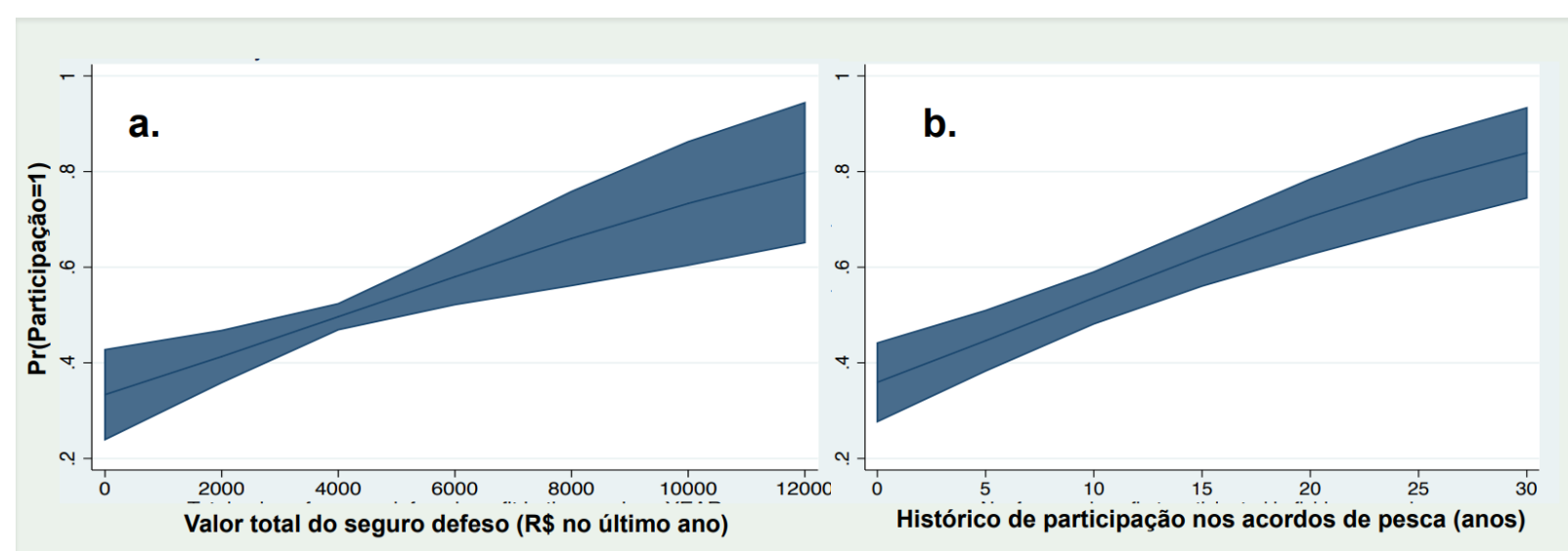

Figura 2. Previsões ajustadas da contribuição marginal para a probabilidade de decisão do domicílio de participar nos esforços de acordo de pesca (calculado nas médias, considerando um intervalo de confiança de 95\%). 
A figura acima mostra a contribuição para a probabilidade de participação do domicílio nos esforços de acordo de pesca na área de estudo em diferentes valores de seguro defeso recebido pelo domicílio (Figura 2.a) e de histórico de sua participação nesses acordos (Figura 2.b). Essa probabilidade é calculada mantendo todas as outras variáveis no seu valor médio. A contribuição marginal nas médias do seguro defeso para a probabilidade de participação do domicílio nos acordos de pesca varia entre $33,1 \%$ a $79,7 \%$, enquanto que a contribuição marginal do histórico de participação varia entre $32,1 \%$ e $80,2 \%$.

\section{Discussão e Conclusão}

Nesse artigo, a proposta foi fazer uma análise sobre os fatores, ou drivers, que determinam a participação dos domicílios da várzea da região do Baixo Amazonas no estado do Pará, nas proximidades da cidade de Santarém, nos esforços de implementação dos acordos de pesca das comunidades. Como resultado da aplicação de um modelo econométrico Probit a dados coletados junto a cerca de 650 domicílios em dois momentos do pulso das águas do rio Amazonas, tem-se que maiores valores do benefício do seguro defeso e histórico mais longo de participação do domicílio contribuem para a decisão de participação nos acordos de pesca. Além disso, a idade do chefe de domicílio, a presença de idosos, o número de pessoas trabalhando fora do domicílio e o valor total das embarcações influenciam aquela decisão, assim como fatores exógenos, como o preço da melancia, da cabeça de gado e da gasolina. Finalmente, a produtividade na atividade da pesca, o número de sítios de pesca visitados e o nível de conflito percebido no período de referência contribuem para a participação nos acordos de pesca.

A importância do seguro defeso em relação à decisão de participar nos acordos de pesca é um tema que merece investigação mais aprofundada, uma vez que esse efeito pode interagir com outras variáveis, como o histórico de participação nos acordos de pesca (que provavelmente carrega um efeito moral para a continuação na participação dos acordos de pesca), ou pode variar conforme o nível de dependência do domicílio em relação ao recurso pesqueiro (JUMBE e ANGELSEN, 2007). No entanto, o efeito do seguro defeso deve ser uma indicação de que esse benefício alivia a restrição orçamentária das famílias da várzea, tendo ele assim um papel social relevante. 
A contribuição da produtividade do domicílio na atividade pesqueira indica que a assistência técnica para as famílias na pesca, mas também em outras atividades produtivas - uma longa reivindicação dos representantes da agricultura familiar - pode ter efeito benéfico sobre a decisão de participação em acordos de pesca e, por sua vez, na manutenção dos estoques pesqueiros.

A análise aqui apresentada contém algumas limitações. Elementos importantes, como o nível de organização social, foram substituídos por proxies, na tentativa de tornar o modelo mais realista. A inclusão de dados sobre as instituições e o nível de organização social, assim como de dados sobre o acesso aos sítios de pesca e sobre a biomassa, certamente tornariam a análise mais qualificada. Esse fator remete à necessidade de um sistema integrado de monitoramento da pesca e das atividades a ela ligadas, assim como do desenvolvimento social e econômico das comunidades e da situação dos recursos pesqueiros para informar as políticas de gestão desses recursos em todos os seus níveis. Sem esse sistema, há grande incerteza sobre os efeitos do comanejo, tanto para o recurso natural quanto para as famílias e domicílios considerados individualmente. Sem incentivos claros, a tentação de violar os acordos pode ser alta.

\section{Agradecimentos}

Esse artigo é um dos resultados do meu trabalho de doutorado e contou com o apoio do projeto da NASA \# NNX12AD27G, dentro do programa deLand-Cover and Land-Use Change, e da Coordenação de Aperfeiçoamento de Pessoal de Nível Superior (Capes). Também colaboraram a Sapopema, a Colônia de Pescadores de Santarém Z20, e a Associação Movimento dos Pescadores do Baixo Amazonas (MOPEBAM). Não posso deixar de agradecer à Dra. Fernanda Nascimento, professora da UFOPA, pelo apoio logístico em Santarém durante os trabalhos de campo, assim como as comunidades que participaram da pesquisa, às famílias entrevistadas que receberam cada pesquisador com carinho e respeito, e à excelente equipe de campo composta por pacientes e perseverantes profissionais e pesquisadores, com os quais aprendi muito. 


\section{Referências}

AGRAWAL, A.; GUPTA, K. Decentralization and participation: the governance of common pool resources in Nepal's Terai. World development, 33(7), 1101-1114, 2005. Link para acesso: www.sciencedirect.com/science/article/pii/S0305750X05000690?via\%3Dihub ALMEIDA, O. Manejo de pesca na Amazônia brasileira. São Paulo: Editora Peirópolis. 2006.

BAYLEY, P. B.; PETRERE, M. Amazon fisheries: assessment methods, current status and management options. Canadian Special Publication of Fisheries and Aquatic Sciences, 385-398, 1989. Link para acesso: www.researchgate.net/publication/284053528

BAYLEY, P. B. Understanding large river: floodplain ecosystems. BioScience, 45(3), 153-158, 1995.1 Link para acesso: www.jstor.org/stable/1312554?Search=yes\&resulttemClick=true\&searchText= Understanding\&searchText=Large \&searchText=River:\&searchText=Floodplain\&search Text=Ecosystems\&searchUri=\%2Faction\%2FdoBasicSearch\%3FQuery\%3DUnders tanding\%2BLarge\%2BRiver\%253A\%2BFloodplain\%2BEcosystems\%26amp\%3Bfilter \%3D\&refreqid=search\%3A9c0a4b2b47128925[ ]e07eb8f3aea11f31\&seq=1\#page scan_tab_contents

BENATTI, J. H. "Proposals, experiences, and advances in the legalization of land tenure in the Várzea."The Amazon Várzea. Springer Netherlands, 67-82, 2011. Link para acesso: link.springer.com/content/pdf/10.1007\%2F978-94-007-0146-5.pdf

CAMERON, A.C.; TRIVEDI, P.K. Microeconometrics: methods and applications. New York: Cambridge University Press. 2005.

CAMERON, A.C.; TRIVEDI, P.K. Microeconometrics using stata (Vol. 2). College Station, TX: Stata press. 2010.

CASTELLO, L.; VIANA, J.P.; WATKINS, G.; PINEDO-VASQUEZ, M.; LUZADIS, V.A. Lessons from integrating fishers of arapaima in small-scale fisheries management at the Mamirauá Reserve, Amazon. Environmental management, 43(2),197-209. 2009. Link para acesso: link.springer.com/article/10.1007/s00267-008-9220-5

CASTRO, F.; MCGRATH, D.G. Moving toward sustainability in the local management of floodplain lake fisheries in the Brazilian Amazon. Human Organization, 62(2), 123-133, 2003. Link para acesso: www.jstor.org/stable/44127340?casa token=irKr1v

DIcsAAAAA:vL5CEwWg29VnsXn_Gejli1ljARolw4EZWuHMDU8JcnY078nfHa7ysvBq6

MOzelwSZ5q08AlynmeY43Eq1iKb68NUsNhnqKD3n-T5t93fUrs-NhLD-

jhO\&seq=1\#page

scan_tab_contents

COULIBALY-LINGANI, P., SAVADOGO, P., TIGABU, M., ODEN, P.C. Factors influencing people's participation in the forest management program in Burkina Faso, West Africa. Forest Policy and Economics, 13(4), 292-302, 2011. Link para acesso:www.sciencedirect.com/science/article/pii/S1389934111000177

DE JANVRY, A.; FAFCHAMPS, M.; SADOULET, E. Peasant household behaviour with missing markets: some paradoxes explained. The Economic Journal, 101(409), 1400-1417, $1991 . \quad$ Link 
para acesso:

AAAA:NM7Is4W4zTGDLZav

Nu3vyILnIpTIRL1j2uaz2y2ZH8LJDCNSBbjHUEA7ZzdaP00gPnJgNHyIXcHs

6iqhvMpJYfjXES5y4C1oEL3TQEThuZ-zKOGuAgjQ\&seq=1\#page

scan_tab_contents

EVANS, L.; CHERRETT,N; PEMSL, D. Assessing the impact of fisheries comanagement interventions in developing countries: A meta-analysis. Journal of environmental management, 92(8), 1938-1949, 2011. Link para acesso: www.sciencedirect.com/science/article/pii/S0301479711000764

GARDNER, R.; OSTROM, E.; WALKER, J.M. The nature of common-pool resource problems. Rationality and society, 2(3), 335-358.1990. Link para acesso: http://dlc.dlib.indiana.edu/dlc/bitstream/handle/10535/670/gardener-the-natureof-common-pool-resource-problems.pdf?sequence=1\&isAllowed=y

GORDON, H. S. The Economic Theory of a Common-Property Resource: The Fishery. The Journal of Political Economy, 124-142,1954. Link para acesso: econ.ucsb.edu/tedb/Courses/Ec100C/Readings/ScottGordonFisheries.pdf

GUTIÉRREZ, N. L.; HILBORN, R.; DEFEO, O. Leadership, social capital and incentives promote successful fisheries. Nature, 470(7334), 386-389, 2011. Link para acesso: www.nature.com/articles/nature09689

HARDIN, G. The Tragedy of the Commons. Science, 161, 1243-1248. 1968. Link para acesso: science.sciencemag.org/content/162/3859/1243

JUMBE, C.B.; ANGELSEN, A. Forest dependence and participation in CPR management: Empirical evidence from forest co-management in Malawi. Ecological Economics, 62(3-4), 661-672, 2007. Link para acesso: www.sciencedirect.com/science/article/pii/S0921800906004113

MCGRATH, D.G.; DE CASTRO, F.; FUTEMMA, C.; DE AMARAL, B.D.; CALABRIA, J. "Fisheries and the evolution of resource management on the lower Amazon floodplain."Human ecology, 21(2), 167-195, 1993. Link para acesso: link.springer.com/article/10.1007/BF00889358

MCGRATH, D.G.; ALMEIDA, O.T.; MERRY, F.D. The influence of community management agreements on household economic strategies: Cattle grazing and fishing agreements on the Lower Amazon floodplain. International Journal of the Commons, 1(1), 67-88, 2007. Link para acesso: dspace.library.uu.nl/handle/1874/29282

MCGRATH, D.G.; CARDOSO, A.; ALMEIDA, O.T.; PEZZUTI, J. Constructing a policy and institutional framework for an ecosystem-based approach to managing the Lower Amazon floodplain. Environment, Development and Sustainability, 10(5), 677-695, 2008. Link para acesso: https://link.springer.com/article/10.1007/s10668-008-9154-3

OSTROM, E. Governing the commons: the evolution of institutions for collective action. New York: Cambridge University Press. 1990.

OVIEDO, A.F.P.; BURSZTYN, M.; DRUMMOND, J.A.. Agora sob nova administração: acordos de pesca nas várzeas da Amazônia Brasileira. Revista Ambiente \& Sociedade, 18(4),119-138, 2015. Link para acesso: 
https://www.researchgate.net/profile/Antonio_Oviedo4/publication/292996319_AGORA SOB_NOVA_ADMINISTRACAO_ACORDOS_DE_PESCA_NAS_VARZEAS_DA AMAZONIA_BRASILEIRA/links/56b50f7a08aebbde1a77c33a.pdf

POMEROY, R.S.; KATON, B.M.;HARKES, I. Conditions affecting the success of fisheries co-management: lessons from Asia. Marine policy, 25(3), 197-208. 2001. Link para acesso: www.sciencedirect.com/science/article/pii/S0308597X01000100

SCHLAGER, E. Common-pool resource theory. Environmental Governance Reconsidered. Cambridge: MIT Press ,145-176, 2004.

SCHONS, S.Z. Forests and fisheries in the Brazilian Amazon: Understanding incentives to comply with conservation efforts. 2017. Tese (Doctor of Philosophy in Forestry) Department of Forest Resources and Environmental Conservation, Virginia Polytechnic Institute and State University, Blacksburg, VA.

SILVA, R.E.da; FERREIRA, R.R. Construção de Acordos de Pesca e Políticas Públicas para gestão de recursos pesqueiros na Região de Santarém, Pará (1990-2004). O Social em Questão, XXI(41), 327-354, 2018. Link para acesso: osocialemquestao.ser.pucrio.br/media/OSQ_41_art_SL2_Silva_Ferreira.pdf 